\title{
Current and Future Intraoperative Imaging Strategies to Increase Radical Resection Rates in Pancreatic Cancer Surgery
}

\author{
Henricus J. M. Handgraaf, ${ }^{1}$ Martin C. Boonstra, ${ }^{1}$ Arian R. Van Erkel, ${ }^{2}$ Bert A. Bonsing, \\ Hein Putter, ${ }^{3}$ Cornelis J. H. Van De Velde, ${ }^{1}$ Alexander L. Vahrmeijer, ${ }^{1}$ and J. Sven D. Mieog ${ }^{1}$ \\ ${ }^{1}$ Department of Surgery, Leiden University Medical Center, Albinusdreef 2, 2300 RC Leiden, The Netherlands \\ ${ }^{2}$ Department of Radiology, Leiden University Medical Center, Albinusdreef 2, 2300 RC Leiden, The Netherlands \\ ${ }^{3}$ Department of Medical Statistics, Leiden University Medical Center, Albinusdreef 2, 2300 RC Leiden, The Netherlands
}

Correspondence should be addressed to Alexander L. Vahrmeijer; a.l.vahrmeijer@lumc.nl

Received 14 March 2014; Revised 6 June 2014; Accepted 20 June 2014; Published 15 July 2014

Academic Editor: Masahiko Hirota

Copyright (C) 2014 Henricus J. M. Handgraaf et al. This is an open access article distributed under the Creative Commons Attribution License, which permits unrestricted use, distribution, and reproduction in any medium, provided the original work is properly cited.

\begin{abstract}
Prognosis of patients with pancreatic cancer is poor. Even the small minority that undergoes resection with curative intent has low 5 -year survival rates. This may partly be explained by the high number of irradical resections, which results in local recurrence and impaired overall survival. Currently, ultrasonography is used during surgery for resectability assessment and frozen-section analysis is used for assessment of resection margins in order to decrease the number of irradical resections. The introduction of minimal invasive techniques in pancreatic surgery has deprived surgeons from direct tactile information. To improve intraoperative assessment of pancreatic tumor extension, enhanced or novel intraoperative imaging technologies accurately visualizing and delineating cancer cells are necessary. Emerging modalities are intraoperative near-infrared fluorescence imaging and freehand nuclear imaging using tumor-specific targeted contrast agents. In this review, we performed a meta-analysis of the literature on laparoscopic ultrasonography and we summarized and discussed current and future intraoperative imaging modalities and their potential for improved tumor demarcation during pancreatic surgery.
\end{abstract}

\section{Introduction}

Surgery is the cornerstone of curative intended treatment of pancreatic cancer [1]. However, resection of pancreatic cancer is only suitable for a minority of patients $[2,3]$. Pancreatic cancer surgery is only conducted when there is a reasonable chance of complete removal of all cancer cells (radical resection), as irradical resection does not improve survival but elicit procedure-related morbidity and mortality [4]. Consequently, pancreatic cancer is known for its high mortality and low 5 -year survival of only $6 \%$ [5].

Despite recent advances in preoperative imaging modalities, such as computed tomography (CT) and magnetic resonance imaging (MRI), the preoperative assessment of resectability is limited due to difficult differentiation of necrosis, fibrosis, and edematous tissue from malignant tumor cells, especially after neoadjuvant therapy [6-9]. The combination of endoscopic ultrasonography (EUS) and laparoscopic ultrasonography improves resectability assessment $[10,11]$. However, microscopic involvement of resection margins ( $\mathrm{R} 1$ resection) is reported up to $75 \%$ of cases, which results in local recurrences and decreased overall survival [12-17]. Therefore, intraoperative imaging strategies accurately visualizing pancreatic cancer cells are highly necessary.

Preoperative imaging of pancreatic cancer using CT, MRI, single-photon emission CT (SPECT), positron emission tomography (PET), and EUS enhances surgical planning, but translating these results to the operating room is difficult due to altered body positioning, tissue manipulation by the surgeon, and lack of sensitivity for subcentimeter lesions. When laparotomy is performed, careful palpation and inspection can yield more information about tumor localization. Minimal-invasive techniques have become important in daily clinical practice but limit tactile feedback. 


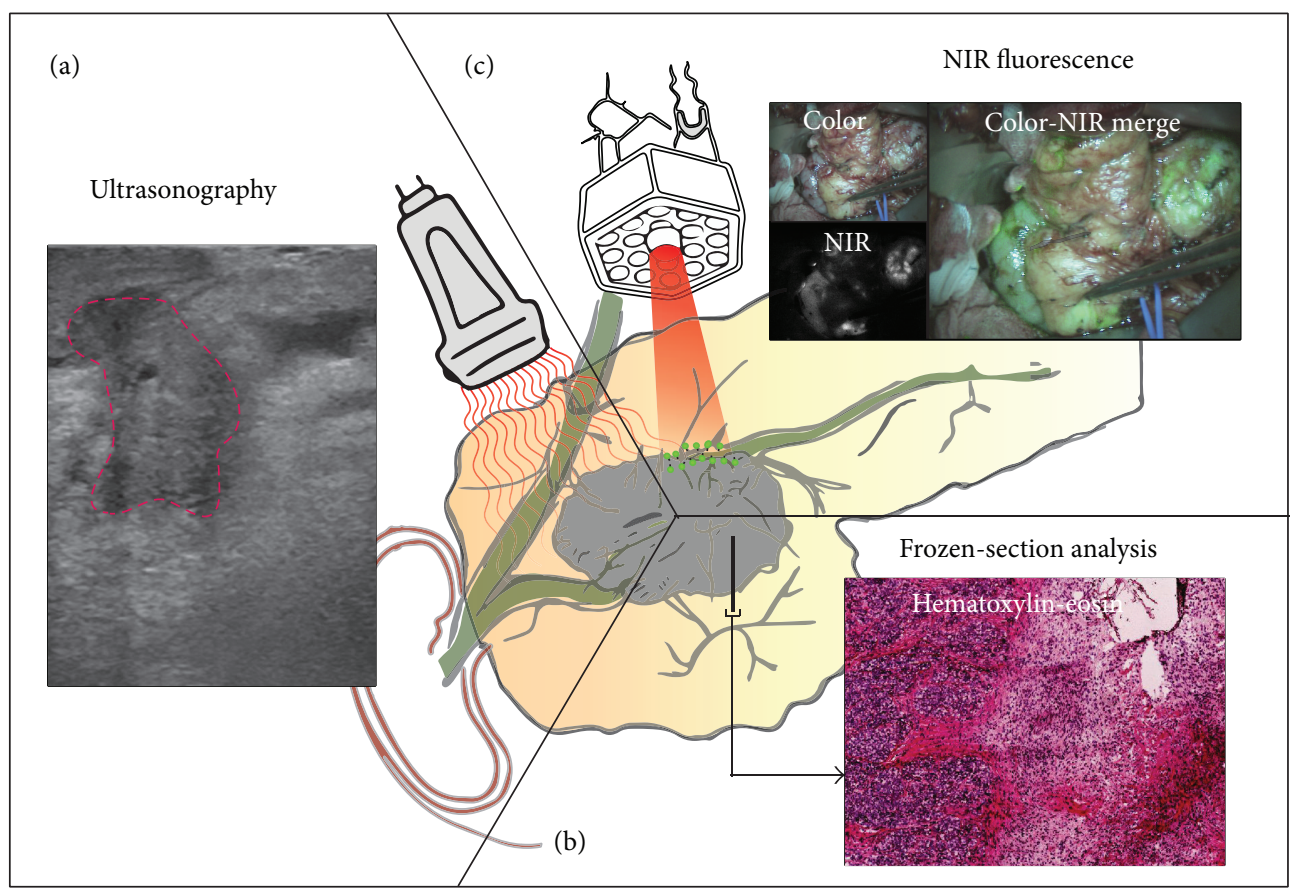

FIGURE 1: Schematic overview of current and future intraoperative imaging modalities. (a) Ultrasonography showing a pancreatic tumor (demarcated with red line). (b) Intraoperative frozen section analysis and (c) optical imaging using near-infrared imaging.

In conclusion, there is a dire need for imaging techniques accurately visualizing and delineating pancreatic cancer during surgery. This review discusses current techniques that are used to assess pancreatic tumor extension during surgery and evaluates the most promising future imaging techniques (Figure 1).

\section{Current Strategies}

2.1. Ultrasonography during Surgery. Ultrasonography (US) is a safe and inexpensive modality that can be used for determination of resectability and identification of metastases (Figure 1(a)) [18-21]. Besides percutaneous application, US is also used during laparoscopy (laparoscopic ultrasonography, LUS) and open surgery (intraoperative ultrasonography, IOUS). Compared to palpation and visual inspection, US is less sensitive for surface evaluations but outperforms in examination of the interior of organs and helps to determine blood flow in vasculature [22]. Its user dependency is a limitation; substantial training and experience are required for generating and interpreting useful images during pancreatic cancer surgery. Furthermore, ultrasound waves are unable to penetrate through air or gas, hampering the visibility of structures and organs located behind hollow organs. But, by slight compression or by imaging from another side, this limitation can mostly be overcome.

Various studies have evaluated the role of LUS in predicting tumor resectability during staging laparoscopy [2339]. The term "resectability" is used to indicate if radical resection $\left(R_{0}\right)$ of the tumor is technically possible in the absence of vascular involvement and distance metastases. Staging laparoscopy combined with LUS is not always used to determine resectability, since it is debated whether this approach should be offered routinely, selectively, or not at all to those who appear resectable during their preoperative workup $[38,40]$. We performed an extensive review of the literature and pooled the available data in a meta-analysis. We included seventeen studies published between 1995 and 2011. We excluded individual patients from the meta-analysis; if patients were diagnosed with unresectable pancreatic cancer during their preoperative workup, but underwent palliative surgery $[27,29,34,36,38]$, patients did not undergo LUS, but only laparoscopy [34, 36, 37]; patients declined surgery $[31,37]$; if patients were diagnosed with other pathology then pancreatic cancer $[31,32,35,37]$. In two studies on selective use of LUS, it was not possible to extract the subpopulation of patients that received LUS assessment [41, 42]. Therefore, these studies were not included. In total, data on 1,255 patients undergoing LUS were available for the metaanalysis. A random effect model was chosen due to significant heterogeneity between studies. Pooled sensitivity of LUS for determining unresectable disease was 76\% (95\% CI $=65-$ $87 \%$ ) and negative predictive value, the proportion of patients correctly diagnosed with resectable disease, was $82 \%(95 \%$ $\mathrm{CI}=75-88 \%$ ) (Figure 2). The variance between studies may partly be explained by a difference in a priori probability, potentially as a result of patient selection by different preoperative imaging modalities. Furthermore, different criteria for unresectability were used.

We were unable to find any study on the use of IOUS in determining resectability, except some outdated literature, which reported success with IOUS in visualizing nonpalpable pancreatic masses [43]. 


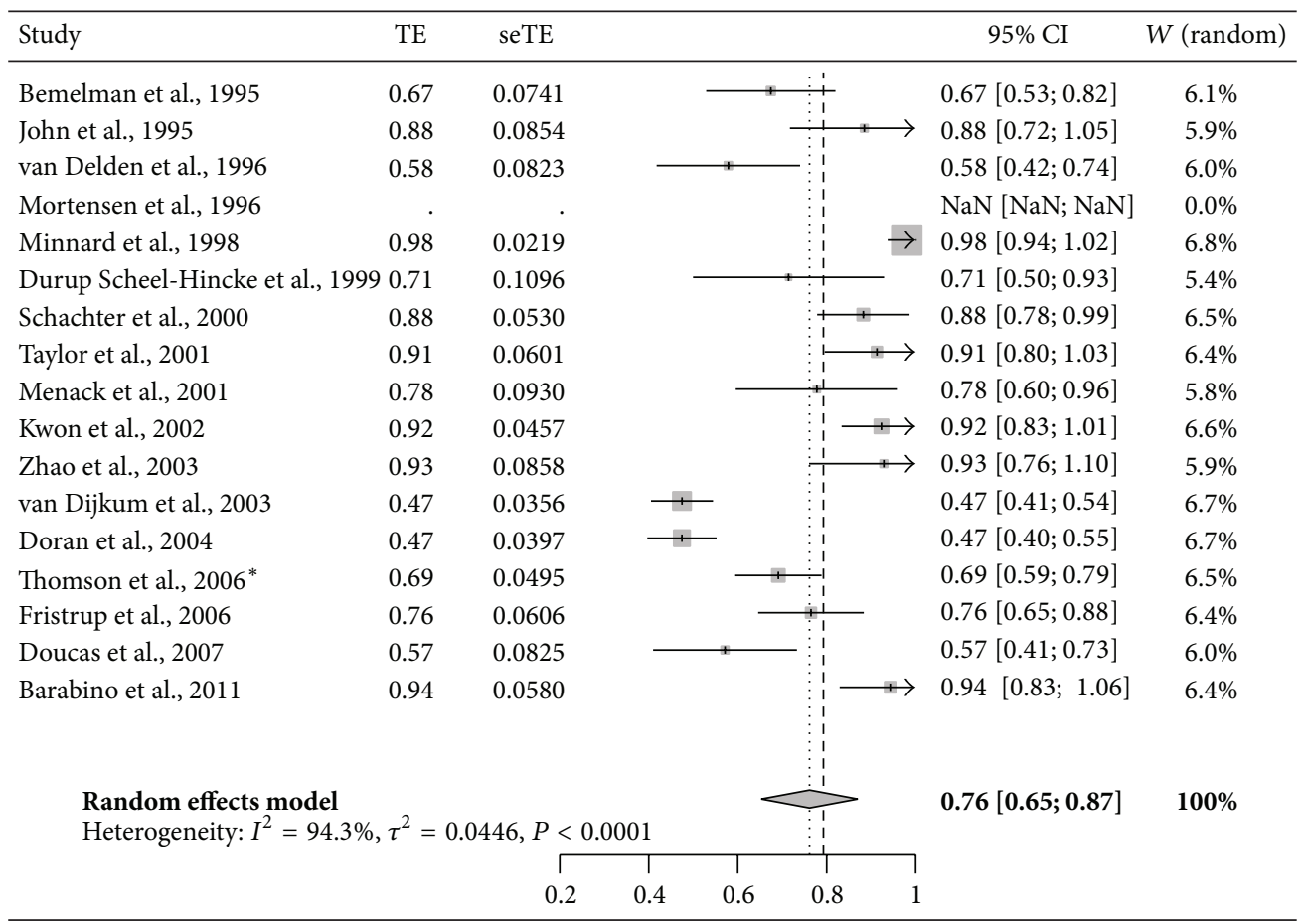

(a)

\begin{tabular}{|c|c|c|c|c|c|}
\hline Study & $\mathrm{TE}$ & seTE & & $95 \% \mathrm{CI}$ & $W$ (random) \\
\hline Bemelman et al., 1995 & 0.65 & 0.0754 & & $0.65[0.50 ; 0.80]$ & $5.7 \%$ \\
\hline John et al., 1995 & 0.79 & 0.1097 & & $0.79[0.57 ; 1.00]$ & $4.3 \%$ \\
\hline van Delden et al., 1996 & 0.78 & 0.0693 & & $0.78[0.64 ; 0.91]$ & $6.0 \%$ \\
\hline Mortensen et al., 1996 & 1.00 & & & 1.00 & $0.0 \%$ \\
\hline Minnard et al., 1998 & 0.98 & 0.0241 & & $0.98[0.93 ; 1.02]$ & $7.7 \%$ \\
\hline Durup Scheel-Hincke et al., 1999 & 0.88 & 0.0781 & & $0.88[0.73 ; 1.04]$ & $5.6 \%$ \\
\hline Schachter et al., 2000 & 0.89 & 0.0510 & & $0.89[0.79 ; 0.99]$ & $6.8 \%$ \\
\hline Taylor et al., 2001 & 0.91 & 0.0613 & & $0.91[0.79 ; 1.03]$ & $6.3 \%$ \\
\hline Menack et al., 2001 & 0.90 & 0.0671 & & $0.90[0.77 ; 1.03]$ & $6.1 \%$ \\
\hline Kwon et al., 2002 & 0.97 & 0.0290 & & $0.97[0.91 ; 1.03]$ & $7.6 \%$ \\
\hline Zhao et al., 2003 & 0.89 & 0.1048 & & $0.89[0.68 ; 1.09]$ & $4.5 \%$ \\
\hline van Dijkum et al., 2003 & 0.74 & 0.0314 & $\longrightarrow$ & $0.74[0.67 ; 0.80]$ & $7.5 \%$ \\
\hline Doran et al., 2004 & 0.80 & 0.0316 & $\div$ & $0.80[0.74 ; 0.87]$ & $7.5 \%$ \\
\hline Thomson et al., $2006^{*}$ & 0.71 & 0.0485 & $\longrightarrow$ & $0.71[0.62 ; 0.81]$ & $6.9 \%$ \\
\hline Fristrup et al., 2006 & 0.84 & 0.0528 & & $0.84[0.73 ; 0.94]$ & $6.7 \%$ \\
\hline Doucas et al., 2007 & 0.42 & $0.0822 \longrightarrow$ & & $0.42[0.26 ; 0.58]$ & $5.4 \%$ \\
\hline Barabino et al., 2011 & 0.88 & 0.0827 & & $0.88[0.71 ; 1.04]$ & $5.4 \%$ \\
\hline \multicolumn{4}{|c|}{$\begin{array}{l}\text { Random effects model } \\
\text { Heterogeneity: } I^{2}=85.9 \%, \tau^{2}=0.0137, P<0.0001\end{array}$} & $0.82[0.75 ; 0.88]$ & $100 \%$ \\
\hline Heterogeneity: $1^{-}=85.9 \%, \tau^{-}=$ & $=0.01$. & , $P<0.0001$ & 1 & & \\
\hline & & 0.2 & 0.6 & & \\
\hline
\end{tabular}

(b)

Figure 2: Forest plot of pooled data on (a) sensitivity and (b) negative predictive value of laparoscopic ultrasonography in predicting unresectability of pancreatic cancer, which is preoperatively considered to be resectable. ${ }^{*}$ Thomson et al. included 152 patients, $61 \%$ had pancreatic adenocarcinoma, $12 \%$ presumed pancreatic cancer, $11 \%$ ampullary cancer, $5 \%$ cholangiocarcinoma, and $11 \%$ had other diagnoses. No data solely describing pancreatic cancer patients was available. 
Besides assessing localization and characteristics of the primary tumor, US can also be used for the detection of previously unnoticed metastases. Sensitivity of laparoscopy combined with LUS reached $100 \%$ in detecting hepatic and peritoneal metastases in a study of 26 patients with pancreatic cancer compared with percutaneous US, CT, or EUS [44].

US is a useful intraoperative imaging technique and provides valuable information about size, localization, and characteristics of lesions. By intraoperative suspicion of unresectability, LUS can aid in avoiding futile resections, and even more when combined with pretherapeutic EUS $[10,11,36,45]$. However, little literature exists about the value of US-guided surgery in reducing positive resection margins in pancreatic cancer surgery.

\subsection{Intraoperative Frozen-Section Analysis. Intraoperative} frozen-section analysis (IFSA) of the margins in the pancreatic neck is commonly performed and currently considered as the most important method for intraoperative assessment of the resection margin (Figure $1(\mathrm{~b})$ ). It is safe, fast and easy to perform; however, it requires significant processing and evaluating time [46]. Additional resection in case of positive resection margins seems logical, but several studies describe no significant survival benefit after reresection [4749]. However, no standardized protocol for frozen sections of pancreatic cancer resection margins was described in the studies. The use of nonstandardized methods for histopathological analysis greatly influences the reporting of resection margin status $[13,17,50]$. This may explain the low sensitivity of only $33 \%$ in evaluating final resection margin status using IFSA [46]. Due to this inconsistent reporting, little is known on the relation between exact tumor location within the pancreas and margin involvement. When standardized protocols are used, IFSA can potentially be a good method for resection margin assessment. However, IFSA will not provide visual and real-time feedback.

\section{Future Strategies}

3.1. Contrast-Enhanced Ultrasonography. Ultrasonography is very usable during pancreatic surgery; hence improvements such as contrast-enhanced US (CEUS) are currently being studied. CEUS uses intravenously administered microbubbles, which allow better determination of vessel infiltration and improved visualization of tumor margins during percutaneous imaging [51-54]. Furthermore, CEUS has already shown to help differentiate between chronic pancreatitis and ductal carcinoma [55]. Finally, CEUS can potentially help in identifying more hepatic metastases [56]. During open resection of colorectal liver metastases, the use of CEUS was of significant value in assessing adequate margins and detecting additional lesions. Preoperative CEUS results are encouraging; translation to the operation room is required to fully study the added value of CEUS during pancreatic cancer surgery.

3.2. Fluorescence-Guided Surgery. Fluorescence-guided surgery has emerged as a novel intraoperative modality to assist surgeons to visualize tumors, sentinel lymph nodes, and vital structures in real time (Figure 1(c)) [57]. Near-infrared (NIR) light (700-900 nanometers) can penetrate through several millimeters tissue, revealing targets below the tissue surface [58]. Consequently, NIR fluorescence imaging is currently a surface technique.

At present only two NIR fluorochromes are FDA approved and can be used in the clinical setting, namely, indocyanine green and methylene blue. Both fluorochromes are nonspecific and are mainly used for sentinel lymph node mapping, bile duct imaging, and ureter visualization [57]. Indocyanine green has been shown to accumulate around hepatic metastasis of pancreatic and colorectal cancers, probably due to retention of indocyanine green in compressed hepatocytes, which is shown by a fluorescent rim $[59,60]$. In $16 \%$ of patients undergoing pancreatic resection without preclinical detected hepatic metastases, fluorescence imaging revealed micrometastases of at least $1.5 \mathrm{~mm}$, which was confirmed by histopathological examination. By revealing undetected hepatic metastases, NIR fluorescence imaging can further decrease the rate of futile pancreatic resections. Furthermore, although its mechanism is unknown, we and others showed that methylene blue tends to accumulate in neuroendocrine tumors, including pancreatic insulinomas $[61,62]$. However, due to the nonspecificity no tumor-specific targeting can be expected of ICG and MB, as was shown by our group in pancreatic carcinomas [63]. To obtain the full advantages of NIR fluorescence imaging for pancreatic cancer visualization, tumor specific NIR conjugated ligands need to be designed and tested.

The biological tumor makeup can be used to visualize pancreatic tumors. In the last decades, research on pancreas carcinoma proteomics gained more attention. An increasing number of differentially expressed proteins are identified (http://www.pancreasexpression.org/). Although very promising, these biomarker studies focus mainly on diagnosis or prevention and not necessarily on biomarkers which can be used to recognize malignant cells and to function as tumor-specific target. Potential biomarker for these approaches must possess additional characteristics, such as homogenic expression, upregulation of more than ten times compared to the surrounding tissue, and localization on the cellular membrane for better accessibility. Ideally, these biomarkers can also recognize precursor lesions at early stages and distinguish between pancreatic cancer and inflammation.

Until now, no membrane-bound biomarkers are validated in the clinic, but recent literature shows very promising results in preclinical studies. Various forms of CEA, integrins, BRCA1, and tumor-associated glycoprotein-17 (TAG17) are overexpressed on pancreatic tumor cells while c-MET, EpCAM, and CXCR-4 are also used as pancreatic cancer stem cell markers $[64,65]$. Biomarkers from the plasmin(ogen) cascade are frequently associated with early stage invasion and cell dissociation [66]. The urokinase receptor (uPAR) is highly upregulated on pancreatic tumors and is associated with tumor invasion and its soluble variant differentiates between pancreatic adenocarcinomas and chronic pancreatitis $[67,68]$. 
Very promising preclinical results are already reported for pancreatic cancer specific molecular targets like CEA, MMPs, claudin-4, RGD, and cholecystokinin-2 receptor [69-73]. The focus within the field is currently shifting towards clinical translation and the first successful in-human results of tumor targeted probes have already been published, although this study is concerning ovarian cancer patients [74]. Real-time NIR fluorescence imaging using tumor-targeted probes has the potential to accurately visualize tumor and its demarcation and hence to increase radical resection rates. The next steps should be clinical translation of pancreatic cancer specific probes, improving commercially available NIR fluorescence imaging systems, and validation of the benefits for patients.

3.3. Nuclear Imaging. Besides fluorophores, ligands can also be conjugated to radiotracers, which are directed to tumorspecific biomarkers eliciting tumor specific signals and enhancing tumor visualization (Figure $1(\mathrm{~d})$ ). These radioactive ligands are used in the preoperative setting with PET and SPECT and intraoperative with radioimmunoguided surgery (RIGS). RIGS was first described in 1984 by Aitken et al., who developed a hand-held gamma detector that can be used intraoperatively, but the technique has become relatively redundant due to the variable sensitivity, the delay in imaging of nearly a week (due to clearance of unbound antibody from the body), and difficulties in handling and disposing the radioactive material [75-78]. A relatively new nuclear imaging technique is freehand SPECT (fSPECT), which was lately introduced as a three-dimensional (3D) imaging and navigation technique that provides real-time images designed for use in the operating room to facilitate detection and resection [79]. However, this technique shows promising results in lymphatic mapping in breast cancer and for the visualization of thyroid diseases but not yet for pancreatic cancer where no known literature exists $[79,80]$.

\section{Discussion}

The field of pancreatic cancer surgery is changing due to improvements in therapies and imaging modalities. These advances have not only led to better pancreatic cancer surgery but also to limitations. The introduction of laparoscopic techniques, for example, has resulted in less postoperative pain, shorter hospital stay, and lower morbidity [81, 82]. However, laparoscopy deprives the surgeon of tactile information, which is helpful during pancreatic cancer surgery. Another example is neoadjuvant therapy, after which a proportion of patients becomes eligible for curative-intended surgery [83]. However, preoperative imaging modalities, such as CT and MRI, drop in sensitivity and specificity in patients who received chemotherapy, for instance, because they cannot accurately distinguish between vascular involvement or vascular encasement only due to periarterial stranding and fibrosis [6-9].

Intraoperative imaging modalities, which can accurately depict pancreatic cancer, can overcome these limitations. Current available technologies, such as US, have their own limitations. US-guided surgery failed to decrease the rate of $\mathrm{R}_{1}$ resections, possibly due to the fact that quality of obtained images is not high enough. The combination of different imaging modalities has proven to be a successful way to overcome separate limitations; PET/CT, for example, fuses anatomical and functional images in a single scan [84]. The combination of US with other techniques could increase functionality. A potential hybrid concept is fSPECT/US, which has already proven to be possible and easy to perform [80].

Molecular imaging is another promising research field where improvements may be expected. NIR fluorescence imaging offers visual guidance during surgery and can therefore potentially reduce the rate of positive resection margins. Fluorescence-guided laparoscopy during hepatopancreatobiliary surgery has already shown its potential to improve intraoperative identification and demarcation of tumors [85]. Remaining fluorescence signal in the resection wound can be an indication of irradical resection, which may make IFSA redundant. To date, no in-human trials have been done with pancreatic cancer specific contrast agents, but preclinical studies are very promising. A major restriction of NIR fluorescence imaging is its limited penetration depth; fluorescence signal is diminished within one centimeter tissue. This is an issue due to the retroperitoneal location of the pancreas. But again, fusing technologies could overcome this limitation. Radiolabeled NIR fluorescence probes may result in the best of two worlds: direct optical guidance and high penetration capacity of the radiotracer [86]. In addition, preoperative planning is possible with PET detection of the radiotracer [87]. Another improvement should be the development of novel probes that are highly specific for pancreatic cancer cells only and hence result in less background signal and lower false-positive rates. Improved fluorophores, which can easily be conjugated to different ligands, are already available, such as ZW800-1 and CW800 [88]. Furthermore, improved imaging systems should become commercially available, making NIR fluorescence imaging available to a broader range of hospitals. But before NIR fluorescence imaging or a hybrid approach can lead to change in patient management, large multicenter studies are necessary to show if these intraoperative imaging modalities are beneficial for patients.

\section{Conclusion}

To improve surgical outcome, reduce irradical resections, and improve patients' survival, novel intraoperative imaging strategies are necessary in pancreatic cancer surgery. Therefore, enhanced imaging technologies that can accurately visualize and delineate pancreatic cancer and its extension in real time are currently being developed and tested. Tumorspecific targeted probes for near-infrared fluorescence imaging are very promising, but research in the next years will have to determine if these modalities are truly of added value for our patients.

\section{Conflict of Interests}

The authors declare that there is no conflict of interests regarding the publication of this paper. 


\section{Authors' Contribution}

Henricus J. M. Handgraaf and Martin C. Boonstra contributed equally to this paper and share the first authorship.

\section{References}

[1] M. K. Diener, H. Knaebel, C. Heukaufer, G. Antes, M. W. Büchler, and C. M. Seiler, "A systematic review and metaanalysis of pylorus-preserving versus classical pancreaticoduodenectomy for surgical treatment of periampullary and pancreatic carcinoma," Annals of Surgery, vol. 245, no. 2, pp. 187200, 2007.

[2] T. Hackert, M. W. Büchler, and J. Werner, "Surgical options in the management of pancreatic cancer," Minerva Chirurgica, vol. 64, no. 5, pp. 465-476, 2009.

[3] K. Y. Bilimoria, D. J. Bentrem, C. Y. Ko, A. K. Stewart, D. P. Winchester, and M. S. Talamonti, "National failure to operate on early stage pancreatic cancer," Annals of Surgery, vol. 246, no. 2, pp. 173-180, 2007.

[4] G. Barugola, S. Partelli, S. Marcucci et al., "Resectable pancreatic cancer: who really benefits from resection?" Annals of Surgical Oncology, vol. 16, no. 12, pp. 3316-3322, 2009.

[5] R. Siegel, D. Naishadham, and A. Jemal, "Cancer statistics, 2013," CA: A Cancer Journal for Clinicians, vol. 63, no. 1, pp. 11-30, 2013.

[6] R. V. Egorov Petrov, E. N. Solodinina, G. G. Karmazanovsky, N. S. Starostina, and N. A. Kuruschkina, "Computed tomographybased diagnostics might be insufficient in the determination of pancreatic cancer unresectability," World Journal of Gastrointestinal Surgery, vol. 5, no. 4, pp. 83-96, 2013.

[7] N. C. Buchs, M. Chilcott, P. Poletti, L. H. Buhler, and P. Morel, "Vascular invasion in pancreatic cancer: imaging modalities, preoperative diagnosis and surgical management," World Journal of Gastroenterology, vol. 16, no. 7, pp. 818-831, 2010.

[8] E. Manak, S. Merkel, P. Klein, T. Papadopoulos, W. A. Bautz, and U. Baum, "Resectability of pancreatic adenocarcinoma: assessment using multidetector-row computed tomography with multiplanar reformations," Abdominal Imaging, vol. 34, no. 1, pp. 75-80, 2009.

[9] C. Cassinotto, J. Cortade, G. Belleannée et al., "An evaluation of the accuracy of CT when determining resectability of pancreatic head adenocarcinoma after neoadjuvant treatment," European Journal of Radiology, vol. 82, no. 4, pp. 589-593, 2013.

[10] M. B. Mortensen, C. W. Fristrup, A. P. Ainsworth, T. Pless, H. O. Nielsen, and C. Hovendal, "Combined preoperative endoscopic and laparoscopic ultrasonography for prediction of RO resection in upper gastrointestinal tract cancer," British Journal of Surgery, vol. 93, no. 6, pp. 720-725, 2006.

[11] M. B. Mortensen, C. Fristrup, A. Ainsworth, H. O. Nielsen, T. Pless, and C. Hovendal, "Combined pretherapeutic endoscopic and laparoscopic ultrasonography may predict survival of patients with upper gastrointestinal tract cancer," Surgical Endoscopy, vol. 25, no. 3, pp. 804-812, 2011.

[12] K. V. Menon, D. Gomez, A. M. Smith, A. Anthoney, and C. S. Verbeke, "Impact of margin status on survival following pancreatoduodenectomy for cancer: the Leeds Pathology Protocol (LEEPP)," HPB, vol. 11, no. 1, pp. 18-24, 2009.

[13] C. S. Verbeke and I. P. Gladhaug, "Resection margin involvement and tumour origin in pancreatic head cancer," British Journal of Surgery, vol. 99, no. 8, pp. 1036-1049, 2012.
[14] T. Schnelldorfer, A. L. Ware, M. G. Sarr et al., "Long-term survival after pancreatoduodenectomy for pancreatic adenocarcinoma: is cure possible?" Annals of Surgery, vol. 247, no. 3, pp. 456-462, 2008.

[15] G. Garcea, A. R. Dennison, C. J. Pattenden, C. P. Neal, C. D. Sutton, and D. P. Berry, "Survival following curative resection for pancreatic ductal adenocarcinoma. A systematic review of the literature," Journal of the Pancreas, vol. 9, no. 2, pp. 99-132, 2008.

[16] I. Esposito, J. Kleeff, F. Bergmann et al., "Most pancreatic cancer resections are R1 resections," Annals of Surgical Oncology, vol. 15, no. 6, pp. 1651-1660, 2008.

[17] C. S. Verbeke, D. Leitch, K. V. Menon, M. J. McMahon, P. J. Guillou, and A. Anthoney, "Redefining the R1 resection in pancreatic cancer," British Journal of Surgery, vol. 93, no. 10, pp. 1232-1237, 2006.

[18] D. Hariharan, V. A. Constantinides, F. E. M. Froeling, P. P. Tekkis, and H. M. Kocher, "The role of laparoscopy and laparoscopic ultrasound in the preoperative staging of pancreaticobiliary cancers-a meta-analysis," European Journal of Surgical Oncology, vol. 36, no. 10, pp. 941-948, 2010.

[19] O. Oshikawa, S. Tanaka, T. Ioka, A. Nakaizumi, Y. Hamada, and T. Mitani, "Dynamic sonography of pancreatic tumors: comparison with dynamic CT," American Journal of Roentgenology, vol. 178, no. 5, pp. 1133-1137, 2002.

[20] M. D’Onofrio, A. Gallotti, and M. R. Pozzi, "Imaging techniques in pancreatic tumors," Expert Review of Medical Devices, vol. 7, no. 2, pp. 257-273, 2010.

[21] S. V. Shrikhande, S. G. Barreto, M. Goel, and S. Arya, "Multimodality imaging of pancreatic ductal adenocarcinoma: a review of the literature," $H P B$, vol. 14, no. 10, pp. 658-668, 2012.

[22] M. Donadon and G. Torzilli, "Intraoperative ultrasound in patients with hepatocellular carcinoma: from daily practice to future trends," Liver Cancer, vol. 2, no. 1, pp. 16-24, 2013.

[23] M. J. Menack, J. D. Spitz, and M. E. Arregui, "Staging of pancreatic and ampullary cancers for resectability using laparoscopy with laparoscopic ultrasound," Surgical Endoscopy, vol. 15, no. 10, pp. 1129-1134, 2001.

[24] W. A. Bemelman, L. T. De Wit, O. M. van Delden et al., "Diagnostic laparoscopy combined with laparoscopic ultrasonography in staging of cancer of the pancreatic head region," British Journal of Surgery, vol. 82, no. 6, pp. 820-824, 1995.

[25] T. G. John, J. D. Greig, D. C. Carter, and O. J. Garden, "Carcinoma of the pancreatic head and periampullary region: tumor staging with laparoscopy and laparoscopic ultrasonography," Annals of Surgery, vol. 221, no. 2, pp. 156-164, 1995.

[26] O. M. van Delden, N. J. Smits, W. A. Bemelman, L. T. de Wit, D. J. Gouma, and J. W. Reeders, "Comparison of laparoscopic and transabdominal ultrasonography in staging of cancer of the pancreatic head region," Journal of Ultrasound in Medicine, vol. 15, no. 3, pp. 207-212, 1996.

[27] M. B. Mortensen, J. D. Scheel-Hincke, M. R. Madsen, N. Qvist, and C. Hovendal, "Combined endoscopic ultrasonography and laparoscopic ultrasonography in the pretherapeutic assessment of resectability in patients with upper gastrointestinal malignancies," Scandinavian Journal of Gastroenterology, vol. 31, no. 11, pp. 1115-1119, 1996.

[28] E. A. Minnard, K. C. Conlon, A. Hoos, E. C. Dougherty, L. E. Hann, and M. F. Brennan, "Laparoscopic ultrasound enhances standard laparoscopy in the staging of pancreatic cancer," Annals of Surgery, vol. 228, no. 2, pp. 182-187, 1998. 
[29] J. Durup Scheel-Hincke, M. B. Mortensen, N. Qvist, and C. P. Hovendal, "TNM staging and assessment of resectability of pancreatic cancer by laparoscopic ultrasonography," Surgical Endoscopy, vol. 13, no. 10, pp. 967-971, 1999.

[30] P. P. Schachter, Y. Avni, M. Shimonov, G. Gvirtz, A. Rosen, and A. Czerniak, "The impact of laparoscopy and laparoscopic ultrasonography on the management of pancreatic cancer," Archives of Surgery, vol. 135, no. 11, pp. 1303-1307, 2000.

[31] A. M. Taylor, S. A. Roberts, and J. M. Manson, "Experience with laparoscopic ultrasonography for defining tumour resectability in carcinoma of the pancreatic head and periampullary region," British Journal of Surgery, vol. 88, no. 8, pp. 1077-1083, 2001.

[32] A. H. Kwon, H. Inui, and Y. Kamiyama, "Preoperative laparoscopic examination using surgical manipulation and ultrasonography for pancreatic lesions," Endoscopy, vol. 34, no. 6, pp. 464-468, 2002.

[33] Z. W. Zhao, J. Y. He, G. Tan, H. J. Wang, and K. J. Li, "Laparoscopy and laparoscopic ultrasonography in judging the resectability of pancreatic head cancer," Hepatobiliary and Pancreatic Diseases International, vol. 2, no. 4, pp. 609-611, 2003.

[34] H. E. Doran, L. Bosonnet, S. Connor et al., "Laparoscopy and laparoscopic ultrasound in the evaluation of pancreatic and periampullary tumours," Digestive Surgery, vol. 21, no. 4, pp. 305-313, 2004.

[35] B. N. J. Thomson, R. W. Parks, D. N. Redhead et al., "Refining the role of laparoscopy and laparoscopic ultrasound in the staging of presumed pancreatic head and ampullary tumours," British Journal of Cancer, vol. 94, no. 2, pp. 213-217, 2006.

[36] C. W. Fristrup, M. B. Mortensen, T. Pless et al., "Combined endoscopic and laparoscopic ultrasound as preoperative assessment of patients with pancreatic cancer," $H P B$, vol. 8, no. 1, pp. 57-60, 2006.

[37] H. Doucas, C. D. Sutton, A. Zimmerman, A. R. Dennison, and D. P. Berry, "Assessment of pancreatic malignancy with laparoscopy and intraoperative ultrasound," Surgical Endoscopy, vol. 21, no. 7, pp. 1147-1152, 2007.

[38] M. Barabino, R. Santambrogio, A. Pisani Ceretti, R. Scalzone, M. Montorsi, and E. Opocher, "Is there still a role for laparoscopy combined with laparoscopic ultrasonography in the staging of pancreatic cancer?" Surgical Endoscopy and Other Interventional Techniques, vol. 25, no. 1, pp. 160-165, 2011.

[39] E. J. N. van Dijkum, M. G. Romijn, C. B. Terwee et al., "Laparoscopic staging and subsequent palliation in patients with peripancreatic carcinoma," Annals of Surgery, vol. 237, no. 1, pp. 66-73, 2003.

[40] D. Stefanidis, K. D. Grove, W. H. Schwesinger, and C. R. Thomas Jr., "The current role of staging laparoscopy for adenocarcinoma of the pancreas: a review," Annals of Oncology, vol. 17, no. 2, pp. 189-199, 2006.

[41] R. White, C. Winston, M. Gonen et al., "Current utility of staging laparoscopy for pancreatic and peripancreatic neoplasms," Journal of the American College of Surgeons, vol. 206, no. 3, pp. 445-450, 2008

[42] C. M. Vollmer, J. A. Drebin, W. D. Middleton et al., "Utility of staging laparoscopy in subsets of peripancreatic and biliary malignancies," Annals of Surgery, vol. 235, no. 1, pp. 1-7, 2002.

[43] M. D. Rifkin and S. M. Weiss, "Intraoperative sonographic identification of nonpalpable pancreatic masses," Journal of Ultrasound in Medicine, vol. 3, no. 9, pp. 409-411, 1984.

[44] J. M. Catheline, R. Turner, N. Rizk, C. Barrat, and G. Champault, "The use of diagnostic laparoscopy supported by laparoscopic ultrasonography in the assessment of pancreatic cancer," Surgical Endoscopy, vol. 13, no. 3, pp. 239-245, 1999.

[45] E. E. Long, J. van Dam, S. Weinstein, B. Jeffrey, T. Desser, and J. A. Norton, "Computed tomography, endoscopic, laparoscopic, and intra-operative sonography for assessing resectability of pancreatic cancer," Surgical Oncology, vol. 14, no. 2, pp. 105-113, 2005.

[46] D. W. Nelson, T. H. Blanchard, M. W. Causey, J. F. Homann, and T. A. Brown, "Examining the accuracy and clinical usefulness of intraoperative frozen section analysis in the management of pancreatic lesions," The American Journal of Surgery, vol. 205, no. 5, pp. 613-617, 2013.

[47] M. Dillhoff, R. Yates, K. Wall et al., "Intraoperative assessment of pancreatic neck margin at the time of pancreaticoduodenectomy increases likelihood of margin-negative resection in patients with pancreatic cancer," Journal of Gastrointestinal Surgery, vol. 13, no. 5, pp. 825-830, 2009.

[48] J. Hernandez, J. Mullinax, W. Clark et al., "Survival after pancreaticoduodenectomy is not improved by extending resections to achieve negative margins," Annals of Surgery, vol. 250, no. 1, pp. 76-80, 2009.

[49] N. L. Lad, M. H. Squires, S. K. Maithel et al., "Is it time to stop checking frozen section neck margins during pancreaticoduodenectomy?" Annals of Surgical Oncology, vol. 20, no. 11, pp. 3626-3633, 2013.

[50] C. S. Verbeke and A. M. Smith, "Survival after pancreaticoduodenectomy is not improved by extending resections to achieve negative margins," Annals of Surgery, vol. 251, no. 4, pp. 776-777, 2010.

[51] M. D’Onofrio, G. Zamboni, N. Faccioli, P. Capelli, and R. Pozzi Mucelli, "Ultrasonography of the pancreas. 4. Contrastenhanced imaging," Abdominal Imaging, vol. 32, no. 2, pp. 171181, 2007.

[52] N. Faccioli, M. D’Onofrio, R. Malagò et al., "Resectable pancreatic adenocarcinoma: depiction of tumoral margins at contrastenhanced ultrasonography," Pancreas, vol. 37, no. 3, pp. 265-268, 2008.

[53] K. Takeda, H. Goto, Y. Hirooka et al., "Contrast-enhanced transabdominal ultrasonography in the diagnosis of pancreatic mass lesions," Acta Radiologica, vol. 44, no. 1, pp. 103-106, 2003.

[54] N. Faccioli, S. Crippa, C. Bassi, and M. D’Onofrio, "Contrastenhanced ultrasonography of the pancreas," Pancreatology, vol. 9 , no. 5 , pp. $560-566,2009$.

[55] K. Koito, T. Namieno, T. Nagakawa, and K. Morita, "Inflammatory pancreatic masses: differentiation from ductal carcinomas with contrast-enhanced sonography using carbon dioxide microbubbles," American Journal of Roentgenology, vol. 169, no. 5, pp. 1263-1267, 1997.

[56] G. Torzilli, D. Del Fabbro, A. Palmisano et al., "Contrastenhanced intraoperative ultrasonography during hepatectomies for colorectal cancer liver metastases," Journal of Gastrointestinal Surgery, vol. 9, no. 8, pp. 1148-1154, 2005.

[57] A. L. Vahrmeijer, M. Hutteman, J. R. van der Vorst, C. J. H. van de Velde, and J. V. Frangioni, "Image-guided cancer surgery using near-infrared fluorescence," Nature Reviews Clinical Oncology, vol. 10, no. 9, pp. 507-518, 2013.

[58] B. Chance, "Near-infrared images using continuous, phasemodulated, and pulsed light with quantitation of blood and blood oxygenation," Annals of the New York Academy of Sciences, vol. 838, pp. 29-45, 1998.

[59] N. Yokoyama, T. Otani, H. Hashidate et al., "Real-time detection of hepatic micrometastases from pancreatic cancer by 
intraoperative fluorescence imaging: preliminary results of a prospective study," Cancer, vol. 118, no. 11, pp. 2813-2819, 2012.

[60] J. R. van der Vorst, B. E. Schaafsma, M. Hutteman et al., "Near-infrared fluorescence-guided resection of colorectal liver metastases," Cancer, vol. 119, no. 18, pp. 3411-3418, 2013.

[61] J. H. Winer, H. S. Choi, S. L. Gibbs-Strauss, Y. Ashitate, Y. L. Colson, and J. V. Frangioni, "Intraoperative localization of insulinoma and normal pancreas using invisible near-infrared fluorescent light," Annals of Surgical Oncology, vol. 17, no. 4, pp. 1094-1100, 2010.

[62] J. R. van der Vorst, A. L. Vahrmeijer, M. Hutteman et al., "Nearinfrared fluorescence imaging of a solitary fibrous tumor of the pancreas using methylene blue," World Journal of Gastrointestinal Surgery, vol. 4, no. 7, pp. 180-184, 2012.

[63] M. Hutteman, J. R. van der Vorst, J. S. D. Mieog et al., "Near-infrared fluorescence imaging in patients undergoing pancreaticoduodenectomy," European Surgical Research, vol. 47, no. 2, pp. 90-97, 2011.

[64] Y. Matsuda, S. Kure, and T. Ishiwata, "Nestin and other putative cancer stem cell markers in pancreatic cancer," Medical Molecular Morphology, vol. 45, no. 2, pp. 59-65, 2012.

[65] T. Wang, S. C. Wentz, N. L. Ausborn et al., "Pattern of breast cancer susceptibility gene 1 expression is a potential prognostic biomarker in resectable pancreatic ductal adenocarcinoma," Pancreas, vol. 42, no. 6, pp. 977-982, 2013.

[66] X. Tan, H. Egami, F. Nozawa, M. Abe, and H. Baba, "Analysis of the invasion-metastasis mechanism in pancreatic cancer: involvement of plasmin(ogen) cascade proteins in the invasion of pancreatic cancer cells," International Journal of Oncology, vol. 28, no. 2, pp. 369-374, 2006.

[67] D. Cantero, H. Friess, J. Deflorin et al., "Enhanced expression of urokinase plasminogen activator and its receptor in pancreatic carcinoma," British Journal of Cancer, vol. 75, no. 3, pp. 388-395, 1997.

[68] Y. Chen, B. Zheng, D. H. Robbins et al., "Accurate discrimination of pancreatic ductal adenocarcinoma and chronic pancreatitis using multimarker expression data and samples obtained by minimally invasive fine needle aspiration," International Journal of Cancer, vol. 120, no. 7, pp. 1511-1517, 2007.

[69] W. Wang, J. Lin, S. Guha et al., "Target-specific agents imaging ectopic and orthotopic human pancreatic cancer xenografts," Pancreas, vol. 40, no. 5, pp. 689-694, 2011.

[70] A. Neesse, A. Hahnenkamp, H. Griesmann et al., "Claudin4-targeted optical imaging detects pancreatic cancer and its precursor lesions," Gut, vol. 62, no. 7, pp. 1034-1043, 2013.

[71] C. Wayua and P. S. Low, "Evaluation of a cholecystokinin 2 receptor-targeted near-infrared dye for fluorescence-guided surgery of cancer," Molecular Pharmaceutics, vol. 11, no. 2, pp. 468-476, 2014.

[72] S. Ji, J. Xu, B. Zhang et al., "RGD-conjugated albumin nanoparticles as a novel delivery vehicle in pancreatic cancer therapy," Cancer Biology and Therapy, vol. 13, no. 4, pp. 206-215, 2012.

[73] S. Kaushal, M. K. McElroy, G. A. Luiken et al., "Fluorophoreconjugated anti-CEA antibody for the intraoperative imaging of pancreatic and colorectal cancer," Journal of Gastrointestinal Surgery, vol. 12, no. 11, pp. 1938-1950, 2008.

[74] G. M. van Dam, G. Themelis, L. M. A. Crane et al., "Intraoperative tumor-specific fluorescence imaging in ovarian cancer by folate receptor- $\alpha$ targeting: first in-human results," Nature Medicine, vol. 17, no. 10, pp. 1315-1319, 2011.
[75] V. di Carlo, F. Badellino, M. Stella et al., "Role of B72.3 iodine 125-labeled monoclonal antibody in colorectal cancer detection by radioimmunoguided surgery," Surgery, vol. 115, no. 2, pp. 190-198, 1994.

[76] M. Stella, P. de Nardi, G. Paganelli et al., "Avidin-biotin system in radioimmunoguided surgery for colorectal cancer: advantages and limits," Diseases of the Colon \& Rectum, vol. 37, no. 4, pp. 335-343, 1994.

[77] D. Sun, M. Bloomston, G. Hinkle et al., "Radioimmunoguided Surgery (RIGS), PET/CT image-guided surgery, and fluorescence image-guided surgery: past, present, and future," Journal of Surgical Oncology, vol. 96, no. 4, pp. 297-308, 2007.

[78] D. R. Aitken, G. H. Hinkle, M. O. Thurston et al., "A gammadetecting probe for radioimmune detection of CEA-producing tumors - Successful experimental use and clinical case report," Diseases of the Colon \& Rectum, vol. 27, no. 5, pp. 279-282, 1984.

[79] T. Wendler, K. Herrmann, A. Schnelzer et al., "First demonstration of 3-D lymphatic mapping in breast cancer using freehand SPECT," European Journal of Nuclear Medicine and Molecular Imaging, vol. 37, no. 8, pp. 1452-1461, 2010.

[80] M. Freesmeyer, T. Opfermann, and T. Winkens, "Hybrid integration of real-time US and freehand SPECT: Proof of concept in patients with thyroid diseases," Radiology, vol. 271, no. 3, pp. 856-861, 2014.

[81] A. C. Jusoh and B. J. Ammori, "Laparoscopic versus open distal pancreatectomy: a systematic review of comparative studies," Surgical Endoscopy and Other Interventional Techniques, vol. 26, no. 4, pp. 904-913, 2012.

[82] A. A. Gumbs, A. M. Rodriguez Rivera, L. Milone, and J. P. Hoffman, "Laparoscopic pancreatoduodenectomy: a review of 285 published cases," Annals of Surgical Oncology, vol. 18, no. 5, pp. 1335-1341, 2011.

[83] M. Herreros-Villanueva, E. Hijona, A. Cosme, and L. Bujanda, "Adjuvant and neoadjuvant treatment in pancreatic cancer," World Journal of Gastroenterology, vol. 18, no. 14, pp. 1565-1572, 2012.

[84] D. W. Townsend, "Dual-modality imaging: combining anatomy and function," Journal of Nuclear Medicine, vol. 49, no. 6, pp. 938-955, 2008.

[85] F. P. R. Verbeek, J. R. Van Der Vorst, B. E. Schaafsma et al., "Image-guided hepatopancreatobiliary surgery using nearinfrared fluorescent light," Journal of Hepato-Biliary-Pancreatic Sciences, vol. 19, no. 6, pp. 626-637, 2012.

[86] S. Lutje, M. Rijpkema, W. Helfrich, W. J. Oyen, and O. C. Boerman, "Targeted radionuclide and fluorescence dual-modality imaging of cancer: preclinical advances and clinical translation," Molecular Imaging and Biology, 2014.

[87] A. J. Beer, H. Kessler, H. J. Wester, and M. Schwaiger, "PET imaging of integrin $\alpha \mathrm{V} \beta 3$ expression," Theranostics, vol. 1, pp. 48-57, 2011.

[88] H. S. Choi, S. L. Gibbs, J. H. Lee et al., "Targeted zwitterionic near-infrared fluorophores for improved optical imaging," Nature Biotechnology, vol. 31, no. 2, pp. 148-153, 2013. 


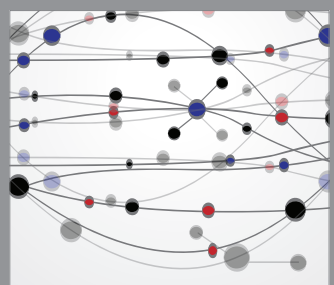

The Scientific World Journal
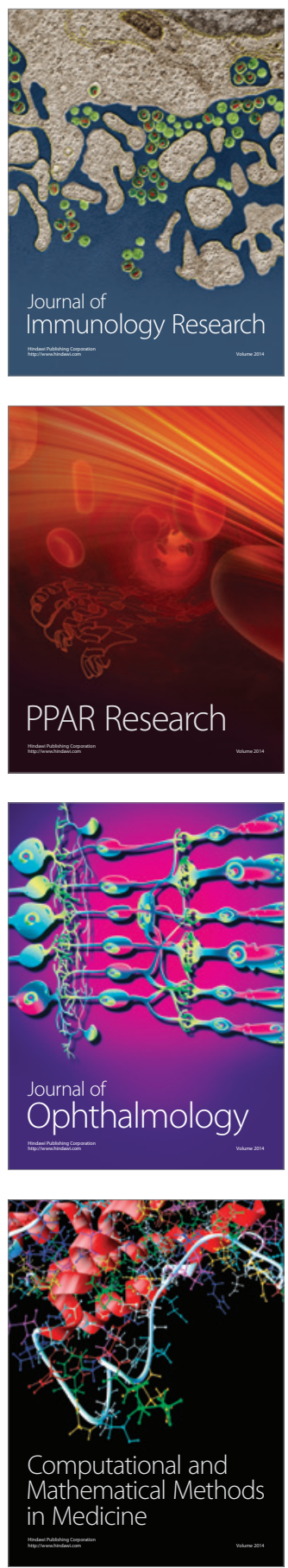

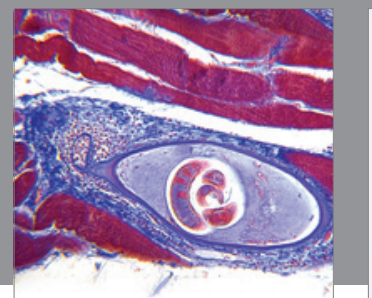

Gastroenterology

Research and Practice
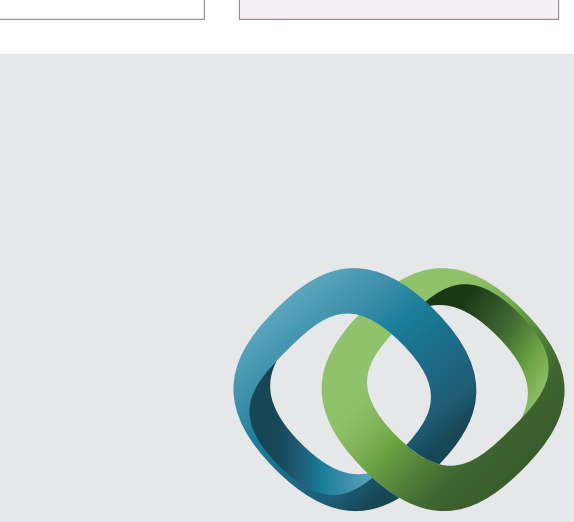

\section{Hindawi}

Submit your manuscripts at

http://www.hindawi.com
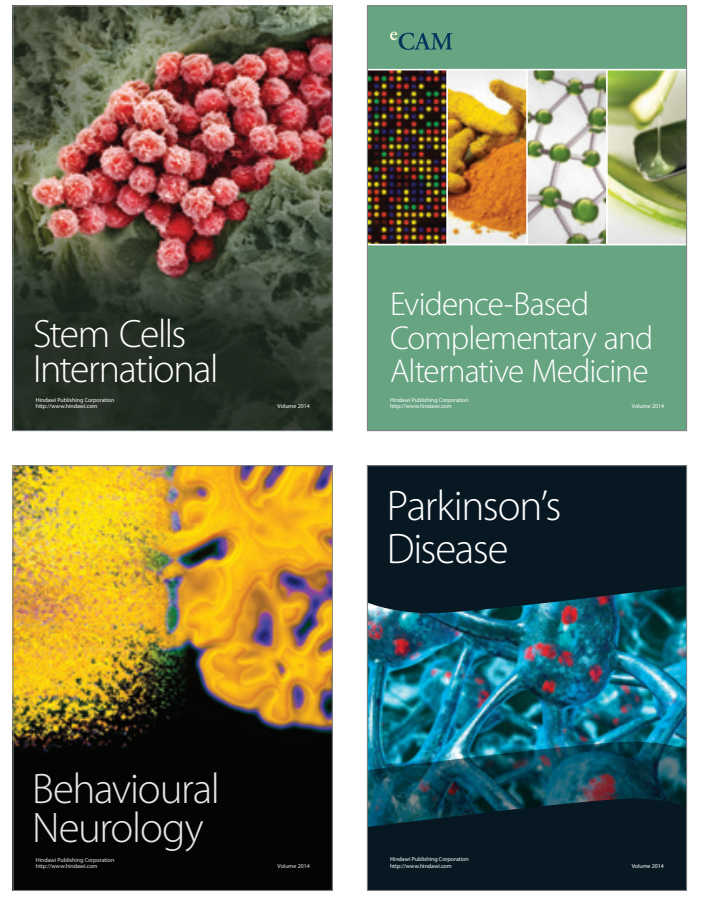
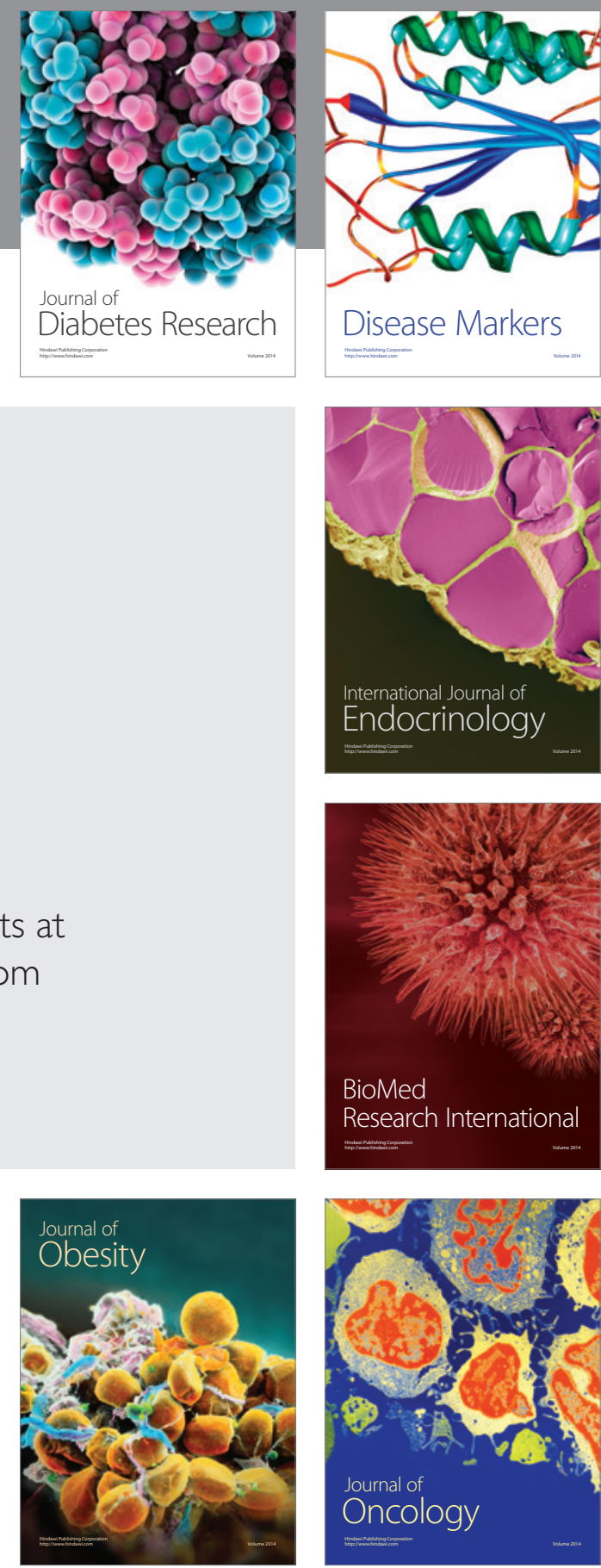

Disease Markers
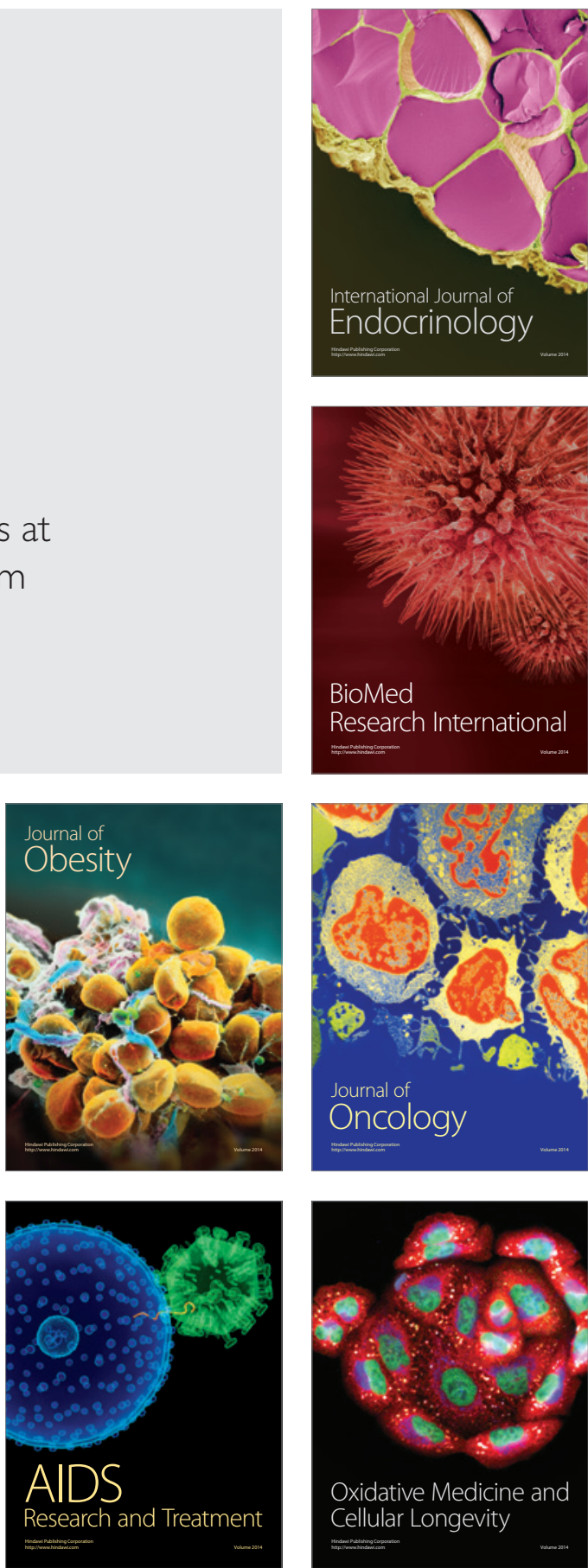